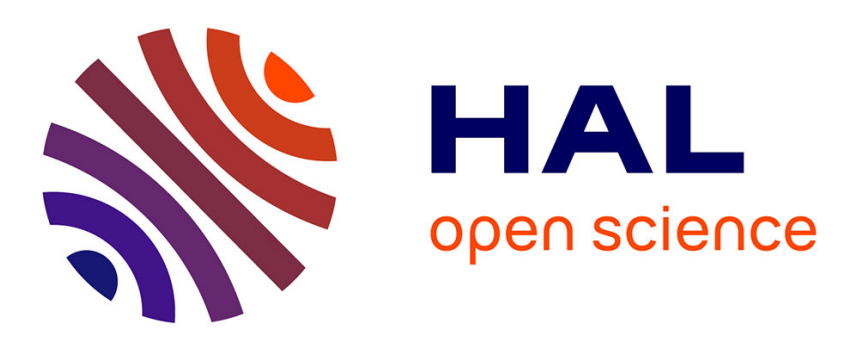

\title{
On the difficulty of using philosophical theories to develop a semantics: the case of Ajdukiewicz
}

Béatrice Godart-Wendling

\section{To cite this version:}

Béatrice Godart-Wendling. On the difficulty of using philosophical theories to develop a semantics: the case of Ajdukiewicz. Piotr Stalmaszczyk (ed.). The Lvov-Warsaw School and Contemporary Philosophy of Language, Brill editions, inPress, Poznań Studies in the Philosophy of the Sciences and the Humanities. hal-03292073

\section{HAL Id: hal-03292073 \\ https://hal.science/hal-03292073}

Submitted on 20 Jul 2021

HAL is a multi-disciplinary open access archive for the deposit and dissemination of scientific research documents, whether they are published or not. The documents may come from teaching and research institutions in France or abroad, or from public or private research centers.
L'archive ouverte pluridisciplinaire HAL, est destinée au dépôt et à la diffusion de documents scientifiques de niveau recherche, publiés ou non, émanant des établissements d'enseignement et de recherche français ou étrangers, des laboratoires publics ou privés. 


\title{
On the difficulty of using philosophical theories to develop a semantics:
}

\section{the case of Ajdukiewicz}

\author{
Béatrice Godart-Wendling \\ Models, Dynamics, Corpora (MoDyCo), CNRS UMR 7114 \\ Paris Lumières University
}

The originality of Ajdukiewicz's approach is to have put forward that the syntactical calculus should be carried out in taking into account the semantical well-formedness of the sentence. Ajdukiewicz writes in his 1935 paper which lays the foundations for his categorial syntax: "It [the problem of syntactic connexion] is concerned with the specification of the conditions under which a word pattern, constituted of meaningful words, forms an expression which itself has a unified meaning (constituted to be sure, by the meaning of the single words belonging to it). A word pattern of this kind is syntactically connected" $(1935 / 1978$, p. 18). This choice of a joint calculus of grammaticality and semantical well-formedness was hailed as particularly innovative (Bach 1988, p. 31-32), since for the first time it did justice to the fact that any speakers assesses simultaneously the grammaticality and the meaning of the statements read or heard. To carry out this syntactico-semantical approach, Ajdukiewicz had two successive sources of inspiration: Husserl's Fourth Logical Research in the thirties and Frege's theory of meaning in the sixties.

The aim of this chapter is to retrace Ajdukiewicz's semantical path by highlighting why these two sources of philosophical inspiration did not allow him to achieve his objective. To show this, we will start by comparing Ajdukiewicz's syntactical and semantical approach with that of his contemporary Rudolf Carnap ${ }^{1}$ who, in the wake of Bertrand Russell's theory of types, had an opposite theoretical position by considering that syntax has to operate on meaningless words. This study, which will show that Ajdukiewicz and Carnap reverse their semantical points of view when they apply their syntax to natural languages, will allow us to understand why Ajdukiewicz looked for another solution by drawing inspiration from Frege's "Sinn und Bedeutung". The analysis of the new conception of meaning propounded by Ajdukiewicz in 1960 will, however, show that it results from a misuse of the Fregean theory. If using philosophical theories to develop a semantics turns out to be a difficult task, Ajdukiewicz's objective would be nevertheless rediscovered and successfully developed in Richard Montague's categorial grammar (1970).

\section{Two opposite approaches of meaning}

\section{I.1. In the framework of formal languages}

Carnap's main idea is that "the aim of logical syntax is to provide a system of concepts, a language, by the help of which the results of logical analysis will be exactly formulable" (1934, p. xiii). Carnap uses this syntactical method to develop two formal languages: Language I that accounts for arithmetic and the Language II, richer in modes of expression, which can state all the propositions of classical physics and mathematics. By working out these languages, Carnac aims to demonstrate that: "the logic of science is nothing other than the logical syntax of the language of science" (ibid.). In part IV of the Logical Syntax

\footnotetext{
1 Ajdukiewicz and Carnap knew each other: Ajdukiewicz refers to Carnap's work in two papers (1934 and 1935) and Carnap quotes Ajdukiewicz in his Logical Syntax (1934, p. 120).
} 
Carnap makes use of the results obtained through the development of languages I and II to propound a general conception of syntax applicable to all languages. Natural verbal languages, however, constitute a special case because their internal logic does not depend exclusively - as in symbolic or scientific languages - on their syntactic structure. Moreover, checking the syntactic adequacy of a natural language is also problematic, since it does not fall within the domain of "pure" syntax, but relies on historical and empirical criteria. Indeed, verbal languages need to answer the question: is this syntax consistent with the speaker's way of speaking this language? Also, although natural languages, because of their complexity, cannot be taken directly into consideration by logical syntax, Carnap nevertheless considers that the development of syntax for formal languages presents the advantage of providing "a system of reference" allowing us to identify the syntactic properties of natural languages $(1934 / 1937$, p. 8). From this point of view Carnap defines the logical syntax in these terms:

"By the logical syntax of a language, we mean the formal theory of the linguistic forms of that language - the systematic statement of the formal rules which govern it together with the development of the consequences which follow from these rules" $(1934 / 1937$, p. 1)

The term of "formal theory" is fundamental, because it implies the rejection of any reference to the meaning of words or to the meaning of propositions. The syntax must only determine the syntactic categories of the words and their order in the sentence. Thus Carnap inscribes his problematic in the continuation of Wittgenstein's thought, since he is directly inspired by the notion of sign as it is expressed in point 3.33 of the Tractatus Logico-Philosophicus:

"In logical syntax the meaning of a sign ought never to play a role; it must admit of being established without mention being thereby made of the meaning of a sign; it ought to presuppose only the description of the expressions." (1921/1999, p.42)

Carnap's definition of syntax - as a system of rules operating on propositions made up of words for which only the category and the order are taken into account - reduces the formal aspect of a language to a "calculus". On the other hand, that words have meaning comes under semantics and gets away from the computational dimension of language.

The main reason advanced by Carnap to justify that syntax has to operate on meaningless words is that words are "inexact" (ungenau). As a result they only allow us to draw conclusions that are themselves "inexact and ambiguous" (1934/1937, p. xv). So, rather than considering the word as a starting point, Carnap adopts the opposite approach of arbitrarily choosing postulates and rules of inference that determine the meaning of the fundamental logical symbols. Carnap therefore advocates a conventionalist methodological position that he explains as follows:

"The whole thing is only a question of the establishment of a convention whose technical efficiency can be discussed" (1934/1937, p. 49)

This free choice concerning the forms of language is claimed by Carnap on the basis of the "principle of tolerance in syntax" that he justifies in these terms:

"In logic, there are no morals. Everyone is at liberty to build up his own logic, i.e. his own form of language, as he wishes. All that is required of him is that, if he wishes to discuss it, he must state his methods clearly, and give syntactical rules instead of philosophical arguments" (1937, p. 52) 
Carnap proposes to reduce words to "characters" (Figur) for which it will not be assumed that they have a meaning or that they designate something, because:

"If logic is to be independent of empirical knowledge, then it must assume nothing concerning the existence of objects" (1937, p. 140)

Concerning the syntactical rules specific to the calculus, Carnap distinguishes "rules of formation" which determine the category and the order of words and allow the construction of propositions, from "rules of transformation" which manage the various inferences that one can make between propositions. These two kinds of rules are illustrated by Carnap thanks to a meaningless sentence:

"For the moment we will leave aside the question of the formal deficiencies of the word-languages, and, by the consideration of examples, proceed to convince ourselves that rules of formation and transformation are of like nature, and that both permit of being formally apprehended. For instance, given an appropriate rule, it can be proved that the word-series "Pirots karulize elastically" is a sentence, provided only that "Pirots" is known to be a substantive (in the plural), "karulize" a verb (in the third person plural), and "elastically" an adverb; all of which, of course, in a well-constructed language - as, for example, in Esperanto - could be gathered from the form of the words alone. The meaning of the words is quite inessential to the purpose, and need not be known. Further, given an appropriate rule, the sentence "A karulizes elastically" can be deduced from the original sentence and the sentence "A is a Pirot" - again provided that the type to which the individual words belong is known. Here also, neither the meaning of the words not the sense of the three sentences need be known" (1937, p. 2-3)

For the rules of formation of Language I, Carnap resorts to gothic writing to represent the syntactical categories of words (variables, predicates, functors, ...) and, in so doing, avoids confusing a word with its syntactical category. Regarding word order, Carnap uses a "language of co-ordinates" such that each word is assigned to a natural number allowing to indicating its position relative to the other words of the sentence. The advantage of such a language of co-ordinates compared to a language which uses - as does Frege - names (in the form of abbreviation) to designate the words of a proposition resides for Carnap in the fact that it eliminates the need to postulate the existence of the object corresponding to the word. It allows Carnap to make the economy of the Axiom of Infinity which asserts the existence of an infinite number of objects. Indeed, a language of co-ordinates simply presupposes that "for every position there is an immediately succeeding one, and that at least one position exists" (1934/1937, p. 141). But it does not establish that there are real objects corresponding to these positions.

The "definition" of words also falls under the rules of formation. Thus, in Languages I and II, a certain number of symbols (the parentheses, the comma, the negation, the disjunction, the conjunction, the symbol of identity, the existential quantifier ...) are called "primitive", in the sense that they do not receive a definition. All other words in the language have an explicit definition formulated through a single sentence, which contains only primitive symbols or words that have been previously defined. These conditions have the effect of establishing an order in the definitions; an order that cannot be changed arbitrarily. In addition, each word belongs to a "chain of definitions" that includes the very definition of the word as well as all the definitions of the words that made up its initial definition. This string of definitions is therefore always finite and unambiguously determined. Each word is thus explicitly defined in Carnap's logical syntax, so that it is possible to eliminate it with the help of its definition. 
Concerning the rules of transformation that show the logical relationships between the propositions, I will only deal with the relation of consequence which is fundamental in Carnap's syntax, because it allows us to determine the meaning of a proposition. For our purposes, it illustrates at the level of the propositions what we have already observed at the level of the word, that is to say that it is possible to carry out a syntactical treatment on units devoid of meaning. Carnap writes:

\begin{abstract}
"The decisive point is the following: in order to determine whether or not one sentence is a consequence of another, no reference need be made to the meaning of the sentences. The mere statement of the truth-values is certainly too little; but the statement of the meaning is, on the other hand, too much. It is sufficient that the syntactical design of the sentences be given. All the efforts of logicians since Aristotle have been directed to the formulation of the rules of inference as formal rules, that is to say, as rules which refer only to the form of the sentences [...]. It is theoretically possible to establish the logical relations (consequence-relation, compatibility, etc.) between two sentences written in Chinese without understanding their sense, provided that the syntax of the Chinese language is given. (In practice this is only possible in the case of the simpler artificially constructed languages)". (1934/1937, p. 258-259)
\end{abstract}

In the same way, Carnap rejects the question regarding the reference of a proposition as irrelevant and even as leading "easily to philosophical pseudo-problems" (1934:1937, p. 195). What is important is that we can distinguish three types of sentence in language:

- The "analytic sentences", such as formulas of logic and mathematics, which are true thanks to their form. They say nothing about the reality but rather intervene in the transformations of propositions that speak about the reality (i.e. the synthetic propositions). - The "contradictory sentences" which are the negation of analytical propositions. They are false by their very form.

- The "synthetic sentences" that speak of the real and whose truth or falsity must be checked through "protocol statements" (i.e. observation statements) of the experimental sciences.

A sentence that does not belong to these three categories is for Carnap meaningless and must be rejected from language.

To determine what a sentence means, we must find all the sentences that are the consequences of this sentence. The "content" (das Gehalt) of a sentence is then defined in Language II by the class of non-analytic sentences which are its consequences. It follows that the terms "content", "sense" and "meaning" are equivalent. From this, Carnap deduces that if two sentences are consequences of one another, they are then equipollent (and vice versa) and therefore synonymous (theorems 34 g.1 and 34 g.2, 1937, p.120). Synonymy is thus dealt with from a syntactical point of view.

Contemporary of Carnap, Ajdukiewicz argues, on the contrary, that syntax has to operate on meaningful words, because language fully participates in "our cognitive process" that is elaborated according to the meanings of a language. Ajdukiewicz does not fail to recall that:

"A language is not unequivocally characterized solely by its vocabulary and the rules of its syntax; it is also characterized by the way meaning is coordinated with its words and expressions" (1934a/1978, p. 39)

The theory, therefore, will have to specify a meaning relationship between the expressions and the syntactic forms (1934a, p. 48). By emphasizing the cognitive role of 
language, Ajdukiewicz defends a strictly conventionalist conception which maintains that our knowledge of the world cannot be directly determined by experience, since it depends on the choice of a language.

More precisely, Ajdukiewicz deals with the meaning of expressions from a dual psychological and logical point of view. At first, he associates - contrary to Carnap - the meaning to a mental act, since he defines it as being:

"Something which decides how, or in what respect, an object must present itself to us for the term to apply to it and which, conversely, is uniquely determined by that aspect" (1931/1978, p. 3)

Thus the object takes part in meaning, but is not reduced to it, because the theory must also be able to account for words - such as verbs - which do not refer to an entity. To do this, Ajdukiewicz will build on this approach to the meaning of terms (taken one by one) by maintaining that a speaker cannot be considered as speaking a certain language if he is not ready, in a given situation, to accept the occurrence of a word inside a meaningful sentence. He writes:

"One uses the word 'table' as an expression of English if one is prepared to accept certain sentences of English in which 'table' occurs and if one is prepared to accept them on the basis of some motives rather than others. If when using 'table' I am prepared to accept sentences like 'A table is a piece of furniture', 'One takes meals at a table', etc. and if, besides, I am prepared to accept the sentence 'This is a table' on the basis of certain intuitive presentations, then I am using 'table' as an expression of English" (1931/1978, p. 33)

This approach to meaning, which relies on context to check the adequate use of the meaning of an expression, is then further understood trough a logical framework that allows for a definition of the meaning of the expressions that is not based on the subjectivity of individual speakers, but rather established relatively to the structure of a language. Ajdukiewicz has however some reservations about the possibility of applying this approach to natural languages, since the meaning of their terms lack precision.

From a logical point of view, a language $L$ is composed for Ajdukiewicz (1934a) of a vocabulary and syntactical rules that are coupled to a matrix containing three kinds of meaning rules:

- The "axiomatic rules of meaning" which specify the sentences that must be accepted unconditionally by all speakers in order to observe the conceptual structure of the language.

- The "deductive rules of meaning" that determine the sentences (i.e. the conclusions) that a person is prepared to accept as soon as he accepts some premises. Modus ponens is an example of these kinds of rules, since a speaker who connects with expressions of $L$ the meaning coordinated with them is prepared to accept the sentence ' $B$ ' as soon as he accepts sentences of the form 'if $A$, then $B$ ' and ' $A$ '.

- The "empirical rules of meaning" which associate certain experience data with sentences that can be accepted by speakers when they are in the presence of these data. Ajdukiewicz's following example allows us to better understand the scope of the rules of meaning:

"If, therefore, English were to be a language of this type with fixed vocabulary, syntax and motivational relations, then in order to speak English, it would be necessary (1) to utter a sentence belonging to English, (2) to be prepared to accept, e.g. the sentence 'Fire!' when seeing a building on fire, (3) to be 
prepared to accept 'Fire!' on the basis of the acceptance of an equivalent sentence, e.g. 'It is burning!', (4) to be prepared to accept the same sentence on the basis of accepting any sentence of the form 'If $C$ has occurred, then it is burning and $C$ has occurred', etc. (5) to be prepared similarly to accept other sentences of English on the basis of suitable motives; the sentences and suitable motives being clearly correlated among themselves" (1931/1978, p. 27).

As Jerzy Giedymin (1978, p. XLV) explains it, analyticity in $L$ corresponds to "the set of sentences dictated by the axiomatic rules of $L$ and their consequences by virtue of deductive rules". Analytic sentences play a determining role for our "conceptual apparatus" because, by structuring it, they make it possible to analyse the phenomena as well as their relations.

In this perspective in which each rule of meaning delimits a set of sentences, two expressions of a language will be synonymous if their substitution does not entail a change of this set (1934a / 1978, p.60). Thus for Ajdukiewicz the meaning of an expression is defined abstractly as well as contextually, corresponding to the positions that an expression can occupy in the matrix.

At this stage, one can observe a tension between the conventionalism of Carnap and his empiricist commitment. Indeed, the first standpoint requires him to choose arbitrarily logical rules and to consider as irrelevant the question of the reference of a sentence. On the other hand, the latter reserves a special status for synthetic propositions - considered by Carnap as the "heart of science" $(1934$, III) - and states that the purpose of a logical system is to allow the analysis of observables (i.e. facts corresponding to the reference of sentences).

\section{I.2. In the framework of natural languages}

In his article entitled "Überwindung der Metaphysik durch logische Analyse der Sprache" (1932), Carnap states the two conditions that a word must fulfil in order to have meaning. The explicit aim of this paper is to show that the words used by metaphysics are meaningless, so that we must prohibit their use in language. Here we find a recurring problematic of the logicist project which, from Frege to Russsell, wants to purify natural language of its imperfections.

The first condition for a word to have meaning is this:

"First the syntax of the word must be fixed, i.e. the mode of its occurrence in the simplest sentence form in which it is capable of occurring; we call this sentence form its elementary sentence. The elementary sentence form for the word 'stone' e.g. is " $x$ is a stone"; in sentences of this form some designation from the category of things occupies the place of ' $x$ ' e.g. 'this diamond', 'this apple"” (1932/1959, p. 62)

The restitution of the elementary sentence allows Carnap to attribute to a word - for which he wants to check the meaning - its "syntactical category" which, in this case, is that "stone" belongs to the category of things. The examples given by Carnap show that this syntactical categorization can go towards an extreme precision, so that it allows us to obtain the complete definition of a word, as well as its chain of definitions. Carnap writes thus about the word "arthropodes":

“ 'arthropodes' are animals with segmented bodies and jointed legs”. Thereby the above-mentioned question for the elementary sentence form of the word 'arthropode', that is for the sentence form 'the thing $\mathrm{x}$ is an arthropode', is 
answered: it has been stipulated that a sentence of this form is deductible from premises of the form ' $x$ is an animal', ' $x$ has a segmented body', ' $x$ has joined legs' and that conversely each of these sentences is deductible from the former sentence. By means of these stipulations about deductibility (in other words: about the truth-condition, about the method of verification, about the meaning) of the elementary sentence about 'arthropode' the meaning of the word 'arthropode' is fixed. In this way every word of the language is reduced to other words and finally to the words which occur in the so-called 'observation sentences' or 'protocol sentences'. It is through this reduction that the word acquires its meaning" (1932/1959, p. 63)

Protocol sentences are sentences that refer to «immediate given». They are the last links in a chain of definitions, and their significance in the theory lies in the fact that they enable Carnap to satisfy his empiricist requirement, even though he recognizes that:

"For our purposes we may ignore entirely the question concerning the content and form of the primary sentences (protocol sentences) which has not yet been definitely settled. In the theory of knowledge it is customary to say that the primary sentences refer to "the given"; but there is no unanimity on the question what it is that is given. [...] Regardless of this diversity of opinion it is certain that a sequence of words has a meaning only if its relations of deductibility to the protocol sentences are fixed, whatever the characteristics of the protocol sentences may be; and similarly, that a word is significant only if the sentences in which it may occur are reducible to protocol sentences." (1932/1955, p. 63)

Determining the meaning of a word belonging to a natural language therefore requires that we restore its syntactical category which allows us to access to its chain of definitions; this chain has to be looked over in order to obtain verifiable observation sentences. This verification requirement is the second and last condition that a word must satisfy when we try to assess whether it actually has a meaning. In the case of formal languages, on the other hand, verification does not always lead to sentences of observation: everything depends on what the language is capable of expressing. If it gives an account of physics, we will of course obtain at the end of the process empirically verifiable sentences, but if it only expresses arithmetic, the verification will consist in following the coherence of a chain of arbitrary definitions.

The calculus of syntactical categories can thus be used by Carnap to denounce the pseudo-propositions, that is to say word sequences containing in meaningful words but whose juxtaposition yields a meaningless sentence. "Caesar is a prime number" is an example of this kind of pseudo-sentence, since "prime number" falls within the category of numbers and therefore cannot be affirmed or denied as a person. As a result, this pseudosentence cannot even be evaluated as a false statement. Carnap deduces that:

"The fact that natural languages allow the formation of meaningless sequences of words without violating the rules of grammar, indicates that grammatical syntax is, from a logical point of view, inadequate. If grammatical syntax corresponded exactly to logical syntax, pseudostatements could not arise. If grammatical syntax differentiated not only the word-categories of nouns, adjectives, verbs, conjunctions etc., but within each of these categories made the further distinctions that are logically indispensable, then no pseudo-statements could be formed. If, e.g. nouns were grammatically subdivided into several kinds of words, according as they designated properties of physical objects, of numbers etc., then the words 
"general" and "prime number" would belong to grammatically different word-categories, and "Caesar is a prime number" would be just as linguistically incorrect as "Caesar is and"." (1932:1955, p. 68)

Establishing a complete agreement between grammatical and logical syntaxes therefore requires that the definitions of words be an integral part of the syntax. In other words, Carnap's logical syntax takes into account word meanings because it must satisfy empirical requirements. Nevertheless, Carnap's theory remains coherent, since he maintains from the beginning that terms such as number, thing or man will be considered as syntactic categories, and that the definition of words will be governed by the rules of sentence formation; rules that are constitutive of syntax.

However if we get out of this system, we may be justified in considering that Carnap's syntactical categories are in fact semantic notions which, through their chain of definitions, receive a lexical definition allowing the calculus of semantic compatibilities (as evidenced by Carnap's analysis of the pseudo-sentence "Caesar is a prime number"). It follows that word meaning that should not be taken into account by logical syntax, since it works on words reduced to "characters", in fact plays a determining role in Carnap's syntactical analysis $^{2}$.

From a different perspective, the problem that Ajdukiewicz seeks to solve is to specify the conditions that a sentence, made up of meaningful words, has to fulfil to have itself a "unified meaning". The idea is therefore to develop a syntactical method that will make it possible to distinguish "syntactically well connected" sentences (as "John loves Mary") from sentences that lack syntactical connection (such as "perhaps horse if will however shine"). In response to the theory of types, Ajdukiewicz chooses to elaborate his concept of "syntactic connection" in the framework of Lesniewski's theory of semantical categories. More precisely, he will retain from this theory the following definition of semantical categories (borrowed from Husserl by Lesniewski):

"Single words and complex expressions of a language can be divided into classes such that two words or expressions belonging to the same class can be substituted for one another, in a context possessing unified meaning, without that context becoming an incoherent word pattern and losing unified sense. On the other hand, two words or expressions belonging to different classes do not possess this property" (1935/1978, p. 118-119)

The concept of semantical category is therefore based on the notion of "substitution". Substitution which allows us to join two words in the same semantical category or to put them into two different categories is carried out by Husserl's "apoditic evidence" which is, as Bar-Hillel stresses, "nothing more than a kind of unsophisticated grammatical intuition" (1967, p. 58). Thus, when Ajdukiewicz illustrates this principle of interchangeability on the sentence "the sun shines", he interchanges "shines" by "burns", "whistles" and "dances" and considers obtaining "true or false sentences which have coherent meaning" (whereas this would not be the case if the substitution took place with such words as "if", "green" or "perhaps"). This "unsophisticated grammatical intuition" therefore simply reiterates the classical parts of speech (the class of verbs, nouns, adjectives, ...). Without relying on explanatory comments, we "feel" that the substitution is possible. Carnap, on the other hand, would have objected that there is no "coherent meaning" in the strings "the sun whistles" or "the sun dances", because their syntactical categories are incompatible, and

\footnotetext{
${ }^{2}$ One could suppose that Carnap has changed his conception of word meaning between his 1932 article and his 1934 book, but this is not the case, since both were written concomitantly in 1932, though The logical Syntax was not published until 1934.
} 
fall - as does the word sequence "Caesar is a prime number" - under a "confusion of spheres" (Shpärenvermengung). The sentences accepted by Ajdukiewicz would therefore be considered as absurd by Carnap, who does not make the distinction, unlike Husserl, between "nonsense" and "absurd", since he puts "Caesar is a prime number" in the same class as "César and is" as shown in the table below:

\begin{tabular}{|l|l|l|}
\hline Examples & \multicolumn{1}{|c|}{ Ajdukiewicz } & \multicolumn{1}{|c|}{ Carnap } \\
\hline Caesar is a prime number & $\begin{array}{l}\text { Syntactically well connected } \\
\text { with a coherent meaning }\end{array}$ & $\begin{array}{l}\text { Error of syntactical category } \\
\text { Confusion of spheres } \\
\text { Absurd }\end{array}$ \\
\hline Caesar and is & Syntactically ill-connected & $\begin{array}{l}\text { Error of syntactical category } \\
\text { Absurd }\end{array}$ \\
\hline The sun shines & $\begin{array}{l}\text { Syntactically well connected } \\
\text { and meaningful }\end{array}$ & Syntactically correct \\
\hline The sun whistles herhaps horse if will \\
however shine & $\begin{array}{l}\text { Syntactically well connected } \\
\text { with a coherent meaning } \\
\text { Devoid of meaning }\end{array}$ & $\begin{array}{l}\text { Error of syntactical category } \\
\text { Confusion of spheres }\end{array}$ \\
\hline John loves Mary & $\begin{array}{l}\text { Syntactically well connected } \\
\text { and meaningful }\end{array}$ & $\begin{array}{l}\text { Error of syntactical category } \\
\text { Absurd }\end{array}$ \\
\hline $\begin{array}{l}\text { The lilac smells strongly } \\
\text { very }\end{array}$ & Syntactically ill-connected & Syntactic error \\
\hline
\end{tabular}

The difference between Ajdukiewicz and Carnap is that the former puts no restriction on the conditions for applying of the substitution, whereas the latter imposes constraints on them thanks to the chains of definitions. Paradoxically, therefore, Ajdukiewicz, who recognizes that words have meaning, calculates no semantical compatibility, whereas Carnap, who maintains that syntax operates on meaningless words, involves the meaning of words. In fact, as Bar-Hillel writes, the concept of semantical category is a "misnomer" that could be replaced with the term "syntactical category":

"Today Lesniewski's term "semantical categories" must be regarded as a misnomer, since the categorization was based on purely syntactical considerations. At the time, however, Lesniewski, like many other authors, believed that well-formedness and meaningfulness are completely coextensive for any proper language" (1967, p. 58)

Ajdukiewicz borrows from Lesniewski's theory the idea that there are two "basic categories" (the sentence $s$ and the name $n$ ) from which one can establish - according to Kotarbinski's term - "categories of functor" which will form an unbounded and ramified ascending hierarchy ${ }^{4}$. Concerning natural language, Ajdukiewicz recognizes that it would require a greater variety of basic categories, and that in particular the category of singular

\footnotetext{
${ }^{3}$ Montague was aware of this problem, since he wrote in English as a Formal Language: "In my semantic categories will be found echoes of the syntactic categories of Ajdukiewicz" (1974/1970, p. 189).

${ }^{4}$ It means that each functorial semantic category is distinct from another and that complex functorial categories are constructed from simpler categories of functors. As a result, this unbounded and ramified ascending hierarchy of functor categories gives raise to an "overabundance of categories" that Emmon Bach (1988, p. 24) denounced. This principle of category construction is related - as Ajdukiewciz points out - with Russell's theory of types: "the system of semantic categories is closely related to the simplified hierarchy of logical types - although ramified to a much higher degree than the latter - and basically constitutes its grammatical semantic counterpart" (1935/1978, p. 119). On this point, see Carnap (1929, p. 30) and Tarski (1933, p. 37).
} 
names should be distinguished from that of universals. We find in Ajdukiewicz's paper (1935) the idea also formulated by Carnap that the construction of a syntax for formal languages, that are relatively poor in means of expression, can in the long term be a precious help to study the formal properties of natural languages.

The main characteristics of Ajdukiewicz's syntax are the following:

1) Each word of a sentence is assigned to a basic or functorial semantic category

2) The categories of functors have the form of a fraction where the denominator indicates the category of dependence of the word, and the numerator the semantical category of the whole expression.

3) The sequence of categories assigned to each word must be reordered according to Polish notation which consists in putting to the extreme left the principal functor followed by its arguments and to reiterate this operation for the secondary functors. This step amounts to classifying the words from the most complex semantical category to the simplest. The advantage of this notation is that it allows us to do without parentheses, since by grouping each functor with its arguments and respecting the hierarchy of operations, it orders the sequence by marking the links of dependence between the categories. It thus avoids any error of syntactical interpretation.

4) Once this sequence of categories is thus ordered, we apply a single rule of simplification that states: if we find, in always reading the sequence from left to right, a fraction whose denominator is identical to the fraction which follows it, then we can simplify these two fractions by replacing them with the numerator of the first fraction.

5 ) If the repeated application of this rule leads to simplifying the category sequence until $s$ is reached, the sentence is syntactically well connected.

In this procedure, the meaning of words intervenes only in step 1, when a semantical category has to be assigned to each word of the sentence. More precisely, this semantical dimension has the form of a semantico-syntactical intuition that speakers would find difficult to explain. Indeed, faced with the sequence "The lilac smells very strongly and the rose blooms", the speaker must know that "lilac" is a name to give it the category $n$, and the calculus of the dependence relationships between words (i.e. the fact that "very" is about "strongly" and not about "the") is done only through intuition. So, this syntax would be inoperative faced with a sequence such as "the lilac smells strongly very" because the speakers would be unable to assign a semantical category to "very". If we consider now all the other steps in Ajdukiewicz's procedure, we see that word meaning plays no role, since we only need to carry out different operations that could be applied to numbers and that a machine could easily run. It is evident that Ajdukiewicz, though he recognizes that words have meaning, in the end cannot include this knowledge in his syntactical algorithm.

\section{Ajdukiewicz and Frege}

Twenty-five years after the development of his categorial syntax, in an unjustly ignored paper entitled "Syntactical Connections between Constituents of Declarative Sentences" (1960), Ajdukiewicz reconsidered the challenge of defining a formalism that would permit integrating the semantical dimension into the syntactical calculus. Although a priori he did not have knowledge of Bar-Hillel's criticism of the notion of semantical category (1953), since his writings never mentioned it, Ajdukiewicz had also become aware of the purely syntactical nature of Husserlian categories, since he wrote:

The semantical categories must be clearly distinguished from the concept of syntactical categories. The term 'semantical category' was introduced for the first time by Husserl; however, the concept he associated with it would correspond better to the term 'syntactical category'. For Husserl pointed out that the expression of a language may be classified according to the role 
they can play within a sentence. He defined, therefore, his categories from the syntactical viewpoint. (1978/1960, p. 275)

With this in mind, Ajdukiewicz proposed the remodelling of his algorithm not only from a semantical, but also syntactical point of view. So, he introduced a new notation to deal with the variety of syntactic structures observed in natural languages (1960: 274). In so doing, he abandoned the idea of proceeding, as for formal languages, according to a purely positional calculus of word order and took into account the impact of inflectional properties of languages on the ordering of words. But while Ajdukiewicz got rid of the framework of formal languages, the new interpretation he chose to give to the notion of semantical category was, however, inscribed in a logical perspective. Indeed, he established his classification according to the "ontological categories of the entities to which linguistic expressions refer" and justified this choice in these terms:

In the logic of language the term 'semantical' is used in a narrower sense to designate all those concepts that are concerned with the relations between linguistic expressions and the entities referred to. This is why a classification of linguistic expressions which reflects ontological categories denoted by them is more appropriately called a classification of semantical categories than one based on syntactical criteria. (1978/1960, p. 275)

But in so doing, Ajdukiewicz made a true theoretical shift towards the position he had defended in 1934, since he had - in the wake of Husserl and Gottlob Frege (1848-1925) supported that:

For such specification of meaning it is not sufficient to fix the correspondence between the words (or expressions) of the language and the objects which, presumably, they denote. The reason for this is two-fold. First, not all expressions designate objects, but only those of a nominal character, i.e. names; nevertheless, all words and expressions of a language have meaning. Second, two expressions can designate the same object and yet have distinct meanings (e.g. the expression 'the highest mountain in Europe' and 'the highest mountain in Switzerland' designate the same object and yet have distinct meaning (1978/1934, p. 39).

Thus, while in 1934 Ajdukiewicz distinguished meaning from reference, his new proposal was to neutralize this opposition by laying down that the meaning of an expression corresponded to its reference. In this framework, he distinguished two basic semantical categories: the category $i$ corresponding to the names of individuals (i.e. the singular terms) that denote some particular objects and the category $w$ of sentences which refer to truth values (that are the "true" or the "false"). By choosing this sentence interpretation, Ajdukiewicz left the Husserlian conception to explicitly endorse the Fregean approach of reference, since he wrote:

Following Frege one assumes in logic that true sentences denote truth while false sentences denote falsity. Truth and falsity are known as 'logical values' (truth-values). The class of sentences may, therefore, be defined from the semantical point of view as that class of linguistic expressions each of which denotes a truth-value. $(1978 / 1960$, p. 275$)$

But this correspondence set by Ajdukiewicz between meaning and reference results from a misuse of Fregean thought. Indeed, Gottlob Frege (1848 - 1925) had clearly distinguished these two concepts in his article "Sinn und Bedeutung" (1892) by arguing that reference was always obtained indirectly through meaning. Thus, the determination of 
the truth value of a sentence was made thanks to its meaning, because it alone could enable us to assess whether the sentence was true or false. It follows from this that by putting a strict correspondence between meaning and reference, Ajdukiewicz no longer offered a model allowing us to understand by what means the reference was reached, but this solution did enable him, however, not to significantly change the model he had proposed in 1935. Indeed, by replacing the old categories $n$ and $s$ (respectively assigned to name and sentence) with the new denominations $i$ and $w$, the generation mode of derived categories could then be retained to represent all the word categories that did not directly realize a referential act. Thus, a first-order predicate, such as "lives" or "sing", was symbolized through a fraction $\frac{w}{i}$ indicating in its denominator that the semantical category of a predicate required the presence of a singular term $i$ in order that a truth value $w$ could be obtained. By so keeping his formalism, Ajdukiewicz therefore succeeded in maintaining the operative nature of the notion of semantical category in his algorithm.

\section{Conclusion}

Depending on their methodological choices and on principles of the syntax, the decision to use (or not) any lexical knowledge from the syntactic level is therefore problematic in the theories of both Carnap and Ajdukiewicz. Indeed, as long as Carnap supports a conventionalist position only, words can be treated as meaningless "characters", but when an empirical verification requirement is added, he is forced to take the meaning of words into consideration. On the other hand, Ajdukiewicz's difficulty lies in the principle of interchangeability which, not constrained semantically, cannot take into account the meaning of words. Carnap will be the first in his Logical syntax (1934/1937, p. 122) to reject this principle of interchangeability and to demonstrate that two words can be "related" (verwandt), that is, interchangeable in certain contexts only (the term of "isogenous" (gattungsgleich) being reserved for the relation of total interchangeability). In this, Carnap will be followed by Chomsky (1963, p. 410 and 1965, p. 83) who will write that interchangeability in context cannot serve as a basic relationship for an adequate grammar of natural languages. Concerning Ajdukiewicz's ontological conception of meaning (1960), it may appear unconvincing to linguists who, rightly, will find it too reductionist. But it did pave the way for a formal calculus of meaning that Montague would complete by giving it, among other things, a stronger theoretical basis thanks to the model theory.

\section{References}

Ajdukiewicz, Kazimierz. 1931. "On the Meaning of Expressions”, in J. Giedymin (ed.) 1978, p. 1-34.

Ajdukiewicz, Kazimierz. 1934a. "Sprache und Sinn”, Erkenntnis IV, p. 100-138. Engl. trans. In J. Giedymin (ed.) 1978, p. 67-89.

Ajdukiewicz, Kazimierz. 1934b. "The World-Picture and the Conceptual Apparatus", in J. Giedymin (ed.) 1978, p. 67-89.

Ajdukiewicz, Kazimierz. 1935. "Die syntaktische Konnexität”, Studia Philosophica I, p. 127. Engl. Trans. in J. Giedymin (ed.) 1978, p. 118-139.

Ajdukiewicz, Kazimierz. 1960. "Syntactical Connexions between Constituents of Declarative Sentences". in J. Giedymin 1978. 269-281.

Ayer, Alfred. (ed.). 1959. Logical Positivism, The Free Press, Glencoe, Illinois.

Bach, Emmon. 1988. "Categorial Grammars as Theories of Language", in Categorial Grammars and Natural Language Structures, Richard T. Oehrle, Emmon Bach \& Deirdre Wheeler (eds.), D. Reidel Publishing Company, p. 17-34. 
Bar-Hillel, Yehoshua. 1967. "Syntactical and Semantical Categories", The Encyclopedia of Philosophy, vol VIII, p. 57-61.

Carnap, Rudolf. 1929. Abriss der Logistik, mit besonderer Berücksichtigung der Relationstheorie und ihrer Anwendungen, Vienne, Julius Springer, Schriften zur wissenschaftlichen Weltauffassung.

Carnap, Rudolf. 1932. "Überwindung der Metaphysik durch logische Analyse der Sprache", Erkenntnis, vol. II. Engl. Transl. « The Elimination of Metaphysics through Logical Analysis of Language », in Ayer (ed.) 1959, p. 60-81.

Carnap, Rudolf. 1934. Die logische Syntax der Sprache. Verlag von Julius Springer, Wien. Engl. Transl. 1937, Logical Syntax, Routledge \& Kegan Paul Ldt, London.

Chomsky, Noam. 1963. "Formal Properties of Grammars", in D. Luce (ed.), Handbook of Mathematical Psychology. John Wiley \& Sons, pp. 323-418.

Chomsky, Noam. 1965. Aspects of the Theory of Syntax, Cambridge, Mass.

Frege, Gottlob. 1892. "Sinn und Bedeutung". Zeitschrift für Philosophie und philosophische Kritik.100. 25-50.

Giedymin, Jerzy (ed.) 1978. Kazimierz Ajdukiewicz: The Scientific World-Perspective and Other Essays, 1931-1963. D. Reidel Publishing Company, Dordrecht.

Godart-Wendling, Béatrice. 1997. "Carnap et Ajdukiewicz: deux conceptions mathématisées de syntaxe", in Le formalisme en question. Le tournant des années 30, Frédéric Nef \& Denis Vernant (ed.), Paris: Vrin.

Husserl, Edmund. 1913. Logische Untersuchungen, 2d sld, vol. 2, Max Niemeyer, Halle.

Montague, Richard. 1970. "English as a Formal Language". Formal Philosophy. Selected Papers of Richard Montague, Richmond H. Thomason (ed.). pp. 188-221. New Haven and London: Yale University Press.

Schlipp, Paul Arthur. 1963. The Philosophy of Rudolf Carnap, Cambridge University Press, La Salle, Illinois.

Simons, Peter M. 1995. "Meaning and Language". The Cambridge Companion to Husserl. Barry Smith \& David Woodruff Smith (eds.). 106-137. Cambridge University Press.

Simons, Peter M. 2001. «Categories, Construction and Congruence: Husserl's Tactics of Meaning ». Grammar in Early Twentieth-Century Philosophy. Ed by Richard Gaskin. 54 - 73. London: Routledge.

Tarski, Alfred. 1933. "The Concept of Truth in Formalized Deductive Sciences", in A. Tarski, Logic, Semantics, Metamathematics, J. Woodger (ed.), Oxford, Oxford University Press, 1956, p. 152-278.

Whitehead Alfred \& Russell, Bertrand. 1910. Principia Mathematica, Cambridge University Press.

Wittgenstein, Ludwig. 1921. Tractatus Logico-Philosophicus, in Annalen der Naturphilosophie. Engl. Transl. in 1999, Mineola, New York: Dover Publications, Inc.

Wolenski, Jan \& Sinisi, Vito (eds.). 1995. The Heritage of Kazimierz Ajdukiewicz, Poznan Studies in the Philosophy of the Sciences and the Humanities, Editions Rodopi B.V. 